\title{
Wake-up Radio-resilient Scanning Mechanism for Mobile Device in IEEE 802.11ba
}

\author{
Hyunhee Park ${ }^{1}$ and Eui-Jik Kim ${ }^{2 *}$ \\ ${ }^{1}$ Department of Computer Software, Korean Bible University, \\ 32 Dongil-ro 214-gil, Nowon-gu, Seoul 01757, South Korea \\ ${ }^{2}$ School of Software, Hallym University, \\ 1 Hallymdaehak-gil, Chuncheon-si, Gangwon-do 24252, South Korea \\ (Received April 6, 2018; accepted May 21, 2018)
}

Keywords: IEEE 802.11 ba, wake-up radio, WUR discovery channel, WUR discovery frame

With the recent spread of the Internet of Things (IoT), smart devices are equipped with a number of sensors to collect data necessary for various applications. A wake-up radio (WUR) is considered a core technology used to overcome the overhead problems of smart devices associated with sensors such as the limited battery lifetime, bandwidth, and inter-related radio range. However, WUR has difficulty adapting a wake-up schedule to unpredictable traffic patterns, which inherently cause communication latency. We propose a WUR discovery frame to carry information for unassociated stations (STAs) to perform WUR-resilient scanning for nearby access points (APs). Furthermore, a WUR discovery channel is proposed to improve location services and roaming purposes.

\section{Introduction}

With the recent spread of the Internet of Things (IoT), smart devices are equipped with a number of sensors to collect data necessary for various applications. Many modern smart devices such as smartphones, tablets, and laptop computers can connect to noncellular networks such as Wi-Fi and Bluetooth, and suffer from the overhead problems associated with sensors such as the limited battery lifetime, bandwidth, and inter-related radio range. ${ }^{(1,2)}$ To overcome the overhead problems of smart devices, the IEEE 802.11ba task group is developing a new low-power wake-up radio (WUR) standard for Wi-Fi networks to reduce remote device power consumption and extend battery life. ${ }^{(2)}$

Users of smart devices know that leaving the device's screen and radio active can drain the battery in a few hours. For example, an iPhone 7 with a $1960 \mathrm{mAh}$ battery can deliver approximately $15 \mathrm{~h}$ of $3 \mathrm{G}$ phone talk time, $12 \mathrm{~h}$ of Internet browsing over an LTE cell connection, or $15 \mathrm{~h}$ of Internet access over Wi-Fi. Short-range Bluetooth radios transmit at 1.0-2.5 mW, but they clearly do not transmit constantly at full power; if they did, the $1960 \mathrm{mAh}$ battery would last no more than 5 or $6 \mathrm{~h}$, even if the phone used no power for anything else. Therefore, the most important challenge is to reduce the power consumption of smart devices. ${ }^{(2)}$ *Corresponding author: e-mail: ejkim32@hallym.ac.kr https://doi.org/10.18494/SAM.2018.1961 
One simple approach is to adopt WUR technology. ${ }^{(3)}$ Stations (STAs) turn off their main radio [i.e., primary connection radio (PCR)] to remain in an idle-listening state using an extremely low power when they are idle. STAs then turn on the PCR to transmit data only when required. This WUR technology represents the ultimate frontier in low-power radio communications. However, it is difficult to adapt the wake-up schedule to unpredictable traffic patterns, which inherently cause communication latency and wake-up without actual communication demands.

In this work, we seek to address this problem by proposing a WUR-resilient scanning mechanism. The proposed mechanism uses a "WUR discovery frame" and a "WUR discovery channel." The former carries essential information for unassociated STAs to perform WURresilient scanning; the latter is a dedicated channel for transmitting the WUR discovery frame. When an unassociated STA does not know in which channel the WUR discovery frames are transmitted, it needs to scan all channels, which can significantly increase the scan latency before discovering new access points (APs). Simulation results show that the proposed WURresilient scanning mechanism outperforms the previous WUR medium access control (MAC) with regard to power consumption by $57 \%$.

\section{System Model}

\subsection{Low-power WUR}

In an ultralow-power design of wireless environment, the most important challenge is to reduce power consumption when STAs are idle. ${ }^{(4)}$ In WUR technology, the main radio is off unless there is something to transmit; then, a WUR wakes up the main radio when there is a WUR packet (WUP) to receive. The main radio transmits and receives user data.

The STAs are put in the sleep mode periodically and only woken up to transmit or receive; this is called the duty-cycled mode, which is a WUR concept. Figure 1 shows an operation of WUR.

(1) In general, an STA in the idle listening state turns the Wi-Fi connection off and only keeps the WUR connection.

(2) When the transmitter has data to send to the receiver, the transmitter sends a WUP to the receiver, requesting that it turns on the Wi-Fi connection.

(3) The receiver internally sends a wake-up signal to the Wi-Fi connection.

(4) The receiver turns on the Wi-Fi connection.

(5) The receiver with Wi-Fi sends a frame (e.g., probe request) to the transmitter to indicate its awake status and the transmitter sends the data to the receiver.

After the association procedure or receiving complete downlink data, a WUR STA may request to enter a WUR mode to reduce power consumption. After receiving confirmation (i.e., WUR mode response) from the AP, the WUR STA enters the WUR mode; this requires explicit WUR mode signaling. If the WUR STA does not request that its 802.11 main radio be turned off, the AP might send WLAN packets to the WUR STA without sending the WUP, which will then be lost. 


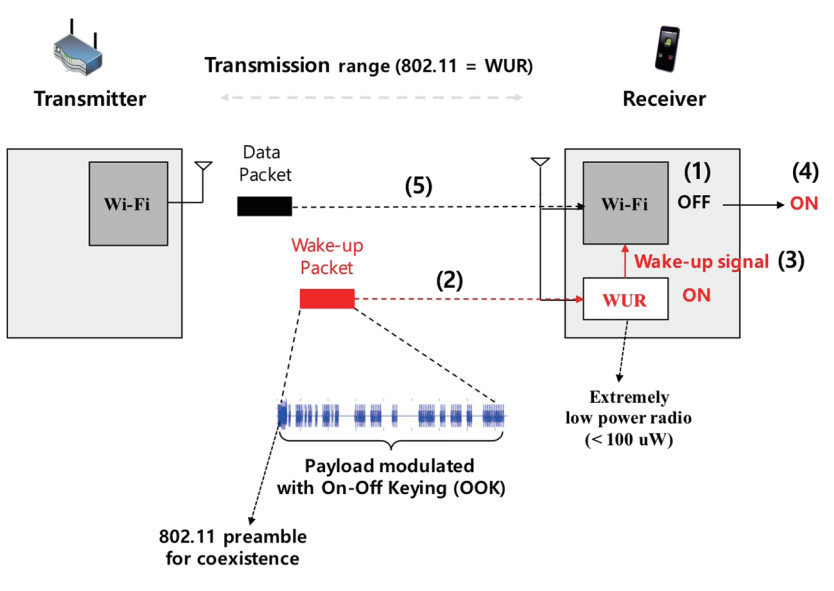

Fig. 1. (Color online) WUR operation.

Whenever the AP wants to send a WLAN packet to the WUR STA in the WUR mode, a WUP should be transmitted in advance to wake up the WUR STA. After transmitting a WUP, the AP should wait for a specific time before sending a WLAN packet. The Wi-Fi main radio turn-on time can be a few milliseconds. Before a WLAN packet transmission, the WUR STA's wake-up can be confirmed through the existing WLAN control signal (e.g., RTS/CTS, PS-Poll).

\subsection{Problem statement of WUR connections}

If the WUR STA is in the idle listening state and the Wi-Fi connection that is turned off in the WUR mode is woken by the wake-up signal, it may lose the connection with the originally connected AP. ${ }^{(5)}$ In particular, this can occur frequently in mobile environments. In this case, even if the Wi-Fi mode is woken by WUR, the WUR STA should scan all channels for the WiFi connection. After all, the WUR STA should take time to scan all channels depending on the number of channels; basically, it will take a long time to scan 10 channels in the Wi-Fi networks.

\section{WUR-resilient Scanning Mechanism}

\subsection{Usage model}

Mobile STAs are scanned as needed for two major reasons: First, mobile STAs sometimes scan through multiple channels for nearby APs and use the measured Wi-Fi signal strength to improve location services. Scanning consumes more power on the PCR than on the WUR. ${ }^{(2)}$ In addition, scanning on the PCR has the risk of conflicting with regular data exchange. In a WUR-facilitated location scan, the mobile STA scans through the channels using the WUR receiver and uses the signal strength measured from WUPs received from adjacent APs to provide additional information to the location services on the mobile STA, meaning that some of the location scans on the PCR can be offloaded to the WUR. The WUR consumes much less power than the PCR; thus, the WUR-facilitated location scan provides an ultralow-power location scan mechanism. 
Second, mobile STAs scan for roaming purposes. These roam scans are sometimes triggered when the link quality degrades. These roam scans take time because they are typically done on multiple channels, and the device either actively or passively scan on each channel. During an active scan, it sends a probe request and typically stays awake until it receives a probe response; during a passive scan, it dwells on each channel for at least a beacon interval to receive a beacon. Thus, scanning through multiple channels introduces roaming latency and consumes power; it sometimes conflicts with regular data exchanges.

In a WUR-facilitated roam scan, the mobile device passively scans through multiple channels using the WUR and collects basic information about nearby APs that can facilitate the STAs' roaming decisions. The WUR consumes much less power than the main radio; thus, the WURfacilitated roam scan provides an ultralow-power roam scan. ${ }^{(5)}$ In addition, owing to the lowpower operation, WUR scans can be performed quite frequently in the background, and thus roaming information can be readily available whenever needed, i.e., it reduces roaming latency.

When an STA is only connected to a fixed home AP, there is no need to frequently scan for connectivity with the AP. ${ }^{(6)}$ However, when the STA used at home is used in other places, it should perform WUR-resilient scanning to find a new AP. If an STA that finds that a Wi-Fi network has been turned off owing to driving arrives at a specific place and wants to make a Wi-Fi connection, the WUR should first be used to determine BSS information about the WiFi connection. Finally, the AP periodically transmits a frame for WUR discovery and the STA first performs WUR-resilient scanning to find the WUR discovery frame.

In WUR-resilient scanning, the WUR on the STA side scans for nearby APs for various purposes such as roaming and location measurements. ${ }^{(7)}$ For this, we define in this paper a WUR discovery frame to carry the essential information for unassociated STAs to perform WUR-resilient scanning. Moreover, when an unassociated STA does not know the channel in which the WUR discovery frames are transmitted, it needs to scan the whole band, which can significantly increase the scan latency before discovering new APs. ${ }^{(1)}$ Therefore, the concept of a WUR discovery channel is proposed for transmitting WUR discovery frames.

\subsection{WUR discovery frame}

For a roaming scan, the STA needs to know the band and channel used by the AP's PCR so that it can switch directly to that particular channel and join the network. The STA also needs to know which AP is being discovered to make better roaming decisions. For a location scan, the STA needs to know the AP's identifier to determine the AP frame on which the measured received signal strength indicator (RSSI) is based. Therefore, we included the following information in the WUR discovery frame as shown in Fig. 2: transmitter ID, main radio

\begin{tabular}{c|c|c|c|c|c|c|}
\hline Type & $\begin{array}{c}\text { Transmitter } \\
\text { ID }\end{array}$ & $\begin{array}{c}\text { Time } \\
\text { Difference } \\
\text { Control }\end{array}$ & $\begin{array}{c}\text { PCR Channel } \\
\text { Information }\end{array}$ & $\begin{array}{c}\text { Primary 20MHz } \\
\text { Channel }\end{array}$ & $\begin{array}{c}\text { Compressed } \\
\text { SSID }\end{array}$ & FCS \\
\hline Octets: & 4 & 12 & 12 & 16 & 16 & 8
\end{tabular}

Fig. 2. WUR discovery frame. 
operation channel information, i.e., operation class and channel number (two octets in total based on the operating channel definition), the primary $20 \mathrm{MHz}$ channel, and compressed SSID. With such information about nearby APs, an STA can decide whether it can associate with a nearby AP without off-channel scanning through the PCR.

An AP includes BSS information in an extended WUP:

(1) The transmitter ID is the BSS identifier in the WUP. It can be BSSID's hash value in a probe request frame for AP's decision of whether to send a probe response. If the transmitter ID value in the broadcast probe request matches the AP's transmitter ID, the AP responds with a probe response.

(2) The time difference control information includes 12-bit synchronization between Wi-Fi and WUR modules.

(3) The PCR channel information includes a one-byte channel class and a one-byte channel number.

(4) The primary $20 \mathrm{MHz}$ channel includes a one-byte channel class and a one-byte channel number.

(5) The compressed SSID is the 16-bit/8-bit hash result of a 48-bit SSID.

The WUR discovery frame can be sent at a different interval from the WUR beacon. The frame interval requirements for synchronization and WUR-resilient scanning may differ.

\subsection{Improvement of scanning latency}

Basically, Wi-Fi uses multiple distinct ranges: $2.4 \mathrm{GHz}, 5 \mathrm{GHz}$, and $900 \mathrm{MHz}$ bands. Each range is divided into multiple channels (e.g., channels $1,6,11,14$, and so forth for a $2.4 \mathrm{GHz}$ Wi-Fi band). When the STA takes $5 \mathrm{GHz}$ in the Wi-Fi network as an example, there is at least $160 \mathrm{MHz}$ of bandwidth through which it has to scan. Depending on the channelization definition in IEEE $802.11 \mathrm{ba}$, each STA needs to scan through many WUR channels to discover new APs and the overall scanning time can be significant. ${ }^{(7)}$ For example, if there is only one $4 \mathrm{MHz}$ channel in each regular $20 \mathrm{MHz}$, then there are eight WUR channels without dynamic frequency selection (DFS) and 19 channels with DFS. Therefore, the STA will need to scan eight times or 19 times at $5 \mathrm{GHz}$, and another three times at $2.4 \mathrm{GHz}$. Furthermore, when IEEE $802.11 \mathrm{ba}$ is allowed in other bands such as $900 \mathrm{MHz}$, then there are even more channels to scan. In addition, when IEEE 802.11ba defines more than one WUR channel for each $20 \mathrm{MHz}$, then there are a significant number of channels to scan through. ${ }^{(1)}$

When the STA takes $5 \mathrm{GHz}$ in the Wi-Fi network as an example, there is at least $160 \mathrm{MHz}$ of bandwidth through which it has to scan. Depending on the channelization definition in IEEE 802.11ba, each STA needs to scan through many WUR channels to discover new APs and the overall scanning time can be significant. ${ }^{(7)}$ For example, if there is only one $4 \mathrm{MHz}$ channel in each regular $20 \mathrm{MHz}$, then there are eight WUR channels without DFS and 19 channels with DFS. Therefore, the STA will need to scan eight times or 19 times at $5 \mathrm{GHz}$, and another three times at $2.4 \mathrm{GHz}$. Furthermore, if IEEE $802.11 \mathrm{ba}$ is allowed in other bands such as $900 \mathrm{MHz}$, then there are even more channels to scan. In addition, if IEEE $802.11 \mathrm{ba}$ defines more than one WUR channel for each $20 \mathrm{MHz}$, then there are a significant number of channels to scan through. ${ }^{(1)}$ 
When a user leaves home and is driving on a road, PCR should be turned off and STA should use a WUR to scan for possible new APs. When the user arrives at a location with Wi-Fi, the WUR needs to discover the existing AP quickly and start to associate with the new AP. ${ }^{(8)}$ After all, defining a WUR discovery frame and defining a limited number of WUR discovery channels allow the WUR to only scan these channels for discovery frames and thus reduce the scanning latency. For example, if we define three WUR discovery channels, then the worstcase latency is reduced from $8 \mathrm{~s}$ on $5 \mathrm{GHz}$ to $3 \mathrm{~s}$ if the WUR discovery frame interval is $1 \mathrm{~s}$, or from 16 to $6 \mathrm{~s}$ if the WUR discovery frame interval is $2 \mathrm{~s}$.

\subsection{Scanning based on advertised discovery channels}

An AP advertises WUR scanning information. Thus, after a user arrives at a location and associates with an AP, it can obtain more information from the PCR regarding the specific WUR operations used by a specific AP or SSID. The STA may store and later recall discovery information, even when the PCR is on. After obtaining such information, the STA can use a WUR to perform a targeted scan in the advertised discovery channel based on specific information such as a compressed SSID. For this, the WUR discovery frame may carry a partial SSID to enhance the scan. In this scenario, the scanning latency can be reduced further from 3 to $1 \mathrm{~s}$.

\subsection{WUR discovery channel}

We define one or a few WUR discovery channels that a WUR transmitter should use when transmitting WUR discovery frames. A WUR AP can select a WUR discovery channel to send out WUR discovery frames.

In the WUR-resilient scanning case, since the Wi-Fi user mobility is low, there is no need to send out frequent WUR discovery frames. In addition, the AP can select a WUR discovery channel to send out WUR discovery frames and there is a maximum PPDU time duration for all WUR discovery frames. Therefore, we do not expect WUR discovery frames to cause congestion in WUR discovery channels.

\section{Simulation Results}

We evaluated the performance of WUR-resilient scanning by conducting extensive simulations. We used PHY/MAC parameters defined in IEEE 802.11ba and 11ax, and we assumed a high-density environment. ${ }^{(1)}$ Moreover, we assumed that mobile devices that support a WUR module were distributed randomly in a square space; APs with WUR and Wi-Fi modules are distributed randomly over the same area. Simulation parameters are summarized in Table 1.

In Fig. 3, the average power consumption decreases as the latency requirement becomes larger. This is because as the latency requirement becomes larger, the STA can increase the waiting time to reduce power consumption. In the WUR-resilient scanning mechanism, each STA can adjust its WUR discovery channel depending on the received WUR discovery 
Table 1

Simulation parameter.

\begin{tabular}{lc}
\hline Parameter & Value \\
\hline WUP length & $500 \mu \mathrm{s}$ \\
WUR power consumption in active state & $100 \mu \mathrm{W}$ \\
WUR active time duration & $2 \mathrm{~ms}$ \\
Channel access delay & $1.5 \mathrm{~ms}$ \\
IEEE 802.11 PHY rate (data/control) & $6.5 \mathrm{Mbps}$ \\
WLAN packet size & $238 \mathrm{bytes}$ \\
Beacon/polling interval & $100 \mathrm{~ms}$ \\
Latency requirement & $100 \mathrm{~ms}$ \\
Bandwidth & $20 \mathrm{MHz}$
\end{tabular}

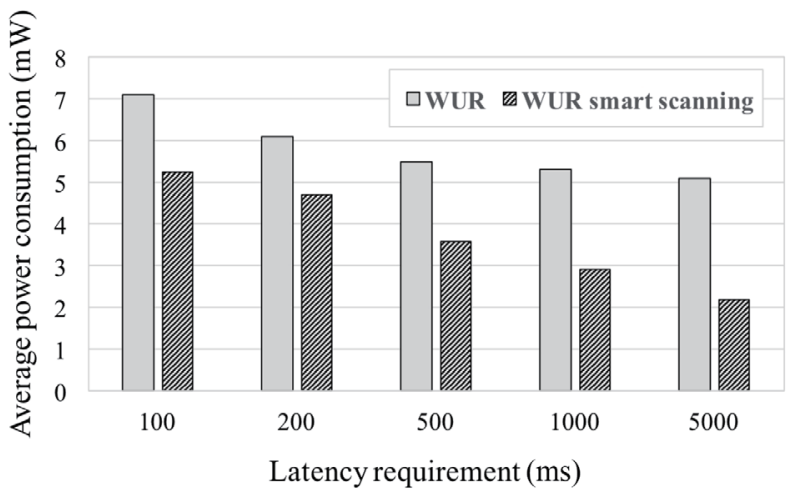

Fig. 3. Average power consumption as a function of latency requirement.

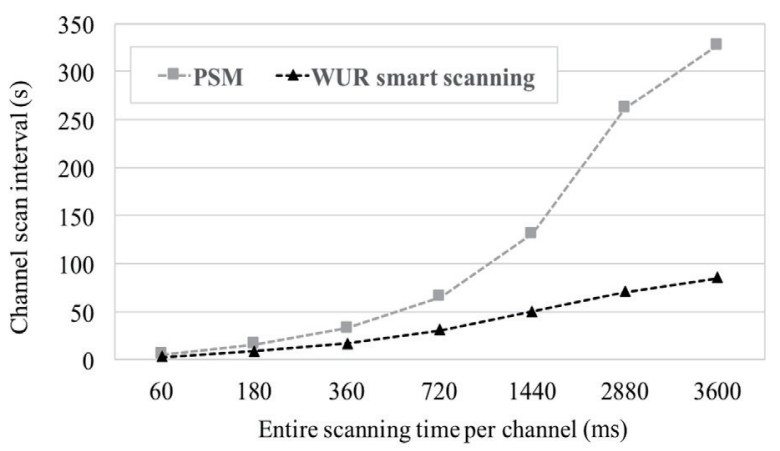

Fig. 4. Channel scan interval as a function of entire scanning time per channel.

frame; therefore, the average power consumption can be remarkably reduced when the latency requirement is larger. Specifically, the WUR-resilient scanning mechanism can reduce the average power by $35 \%$ for a $500 \mathrm{~ms}$ latency requirement and $57 \%$ for a $5000 \mathrm{~ms}$ latency requirement compared with the existing WUR mechanism.

In addition, we carried out simulations to investigate the effect of the channel scan interval on the entire scanning time per channel. We assume that there are 11 channels. For example, a $16 \mathrm{~ms}$ scanning is entirely performed for 11 channels so that the entire scanning time is calculated as $180 \mathrm{~ms}$. From Fig. 4, the channel scan interval apparently increases proportionally with the entire scanning time per channel because the channel scan interval increases as the scanning time per channel increases. In the conventional power saving mode (PSM), the channel scan interval increases significantly as the scanning time per channel increases; in contrast, it increases linearly in the proposed WUR-resilient scanning mechanism. Essentially, when the channel scan interval increases, the WUR-resilient scanning mechanism can adjust the entire scanning time per channel depending on the information of the WUR discovery frame received by near APs.

Consequently, it can be concluded that the WUR-resilient scanning mechanism is a better solution than the existing WUR mechanism in terms of power saving using the WUR discovery frame and WUR discovery channels. 


\section{Conclusions}

The WUR can reduce the power consumption and extend the battery life of smart devices equipped with sensors. However, the WUR has difficulty adapting a wake-up schedule to unpredictable traffic patterns. Thus, in this paper, we present a WUR-resilient scanning mechanism. In WUR-resilient scanning, the WUR at the STA side scans for nearby APs for various purposes such as roaming and location measurements. In this paper, we define a WUR discovery frame to carry the essential information for unassociated STAs to perform WURresilient scanning. For roaming scan, the STA needs to know the band and channel used by the AP's PCR so that it can switch directly to a particular channel to join the network. The STA also needs to know which AP is being discovered to make better roaming decisions. For a location scan, the STA only needs to know the AP identifier to determine on which AP frame the measured RSSI is based. In conclusion, we defined one or a few WUR discovery channels that a WUR transmitter should use to transmit WUR discovery frames. A WUR AP can select one WUR discovery channel to send out WUR discovery frames.

\section{Acknowledgments}

This research was supported by Hallym University Research Fund, 2018 (HRF-201801-013).

\section{References}

1 R. Piyare, A. L. Murphy, C. Kiraly, P. Tosato, and D. Brunelli: IEEE Commun. Surv. Tutorials 19 (2017) 2117. https://doi.org/10.1109/IEEESTD.2017.8053470

2 D. K. McCormick: Proc. IEEE Standards (IEEE, 2017) 1. https://doi.org/10.1109/IEEESTD.2017.8055459

3 K. W. Choi, L. Ginting, P. A. Rosyady, A. A. Aziz, and D. I. Kim: IEEE Trans. Wireless Commun. 16 (2017) 221. https://doi.org/10.1109/TWC.2016.2621766

4 P. Le-Huy and S. Roy: Mobile Networks Appl. 15 (2010) 226. https://doi.org/10.1007/s11036-009-0184-3

5 Y. Kondo, H. Yomo, S. Tang, M. Iwai, T. Tanaka, H. Tsutsui, and S. Obana: Comput. Commun. 35 (2012) 1725. https://doi.org/10.1016/j.comcom.2012.04.022

6 H. Yomo, Y. Kondo, N. Miyamoto, S. Tang, M. Iwai, and T. Ito: Proc. IEEE GLOBECOM 2012 (IEEE, 2012) 5206. https://doi.org/10.1109/GLOCOM.2012.6503947

7 T. Yoshiwaka, H. Yomo, and T. Ito: Proc. IEEE 79th Vehicular Technol. Conf. (IEEE, 2014) 1. https://doi. org/10.1109/VTCSpring.2014.7023144

8 S. Basagni, V. D. Valerio, G. Koutsandria, C. Petrioli, and D. Spenza: Proc. 14th Int. Conf. Mobile Ad Hoc and Sensor Systems (IEEE, 2017) 257. https://doi.org/10.1109/MASS.2017.18 